\title{
Activation of Human Cerebral and Cerebellar Cortex by Auditory Stimulation at $40 \mathrm{~Hz}$
}

\author{
Maria A. Pastor, ${ }^{1}$ Julio Artieda, ${ }^{1}$ Javier Arbizu, ${ }^{2}$ Josep M. Marti-Climent, ${ }^{2}$ Ivan Peñuelas, ${ }^{2}$ and \\ Jose C. Masdeu ${ }^{1}$ \\ Departments of ${ }^{1}$ Neurology and ${ }^{2}$ Nuclear Medicine, University of Navarra School of Medicine, 31080 Pamplona, Spain
}

We used functional brain imaging with positron emission tomography (PET)- $\mathrm{H}_{2}{ }^{15} \mathrm{O}$ to study a remarkable neurophysiological finding in the normal brain. Auditory stimulation at various frequencies in the gamma range elicits a steady-state scalp electroencephalographic (EEG) response that peaks in amplitude at $40 \mathrm{~Hz}$, with smaller amplitudes at lower and higher stimulation frequencies. We confirmed this finding in 28 healthy subjects, each studied with monaural trains of stimuli at 12 different stimulation rates $(12,20,30,32,35,37.5,40,42.5,45$, $47.5,50$, and $60 \mathrm{~Hz}$ ). There is disagreement as to whether the peak in the amplitude of the EEG response at $40 \mathrm{~Hz}$ corresponds simply to a superimposition of middle latency auditory evoked potentials, neuronal synchronization, or increased cortical synaptic activity at this stimulation frequency. To clarify this issue, we measured regional cerebral blood flow (rCBF) with $\mathrm{PET}-\mathrm{H}_{2}{ }^{15} \mathrm{O}$ in nine normal subjects at rest and during auditory stimulation at four different frequencies $(12,32,40$, and $47 \mathrm{~Hz}$ ) and analyzed the results with statistical parametric mapping. The behavior of the rCBF response was similar to the steadystate EEG response, reaching a peak at $40 \mathrm{~Hz}$. This finding suggests that the steady-state amplitude peak is related to increased cortical synaptic activity. Additionally, we found that, compared with other stimulation frequencies, $40 \mathrm{~Hz}$ selectively activated the auditory region of the pontocerebellum, a brain structure with important roles in cortical inhibition and timing.

Key words: steady-state auditory evoked potentials; gamma oscillatory activity; regional cerebral blood flow; positron emission tomography; cerebellum; auditory cortex
In humans, auditory stimulation at different gamma frequencies elicits an electroencephalographic (EEG) steady-state response (SSR) that cycles at the stimulation frequency and has the greatest amplitude when the stimulus is given at $40 \mathrm{~Hz}$ (Galambos et al., 1981). Lower or higher frequencies produce a response of smaller amplitude. These oscillatory responses seem to be generated at the level of the auditory cortex, although modulated by thalamocortical systems (Makela and Hari, 1987; Steriade et al., 1991). The significance and origin of the steady-state potentials continue to be debated (Basar et al., 1987; Santarelli et al., 1995; Gutschalk et al., 1999). It is unclear whether the power increment of the steady-state auditory response at $40 \mathrm{~Hz}$ results merely from the temporal coherence (phase summation) of "middle latency" evoked responses, phase synchronization of a pool of cortical neurons, or a true increase in cortical synaptic activity at $40 \mathrm{~Hz}$. Synaptic activity causes an increment in regional cerebral blood flow $(\mathrm{rCBF})$. To test the hypothesis that the enhanced response at $40 \mathrm{~Hz}$ reflects increased synaptic cortical activity, we measured rCBF with positron emission tomography (PET) in the auditory cortex and other brain regions during auditory stimulation at different frequencies. A rise in $\mathrm{rCBF}$ at $40 \mathrm{~Hz}$ stimulation would suggest that the enhanced EEG response corresponds to increased synaptic activity at this frequency.

Received June 11, 2002; revised Sept. 6, 2002; accepted Sept. 23, 2002.

This work was supported by the University of Navarre Research Fund PIUNA 13298312 and the Spanish Ministerio de Educación y Cultura Direccion General de Investigacion Cientifica y Tecnica PB 92-0713. We thank Profs. David Brooks and Richard Wise for help with preliminary data analysis, Dr. John Ashburner for reviewing the methodological aspect of this study, and Prof. Karl Friston for his valuable suggestions on this manuscript.

Correspondence should be addressed to Dr. Maria A. Pastor, Department of Neurology, University of Navarra School of Medicine, Clinica Universitaria, 31080 Pamplona, Spain. E-mail: mapastor@unav.es.

Copyright (C) 2002 Society for Neuroscience 0270-6474/02/2210501-06\$15.00/0
Most studies of oscillatory behavior have concentrated on the activity of the cortex and thalamus. PET allowed us to study the effect of gamma frequency stimulation on other brain structures, to test a second hypothesis: namely, that given the singular behavior of the EEG response to auditory stimulation at $40 \mathrm{~Hz}$, brain regions outside the auditory cortex are activated specifically by stimulation at $40 \mathrm{~Hz}$ and not at other gamma frequencies.

\section{MATERIALS AND METHODS}

Subjects. We studied 28 normal volunteers (16 females; 12 males; mean age $=35.3 ; \mathrm{SD}=4.2$ ) who had no history of audiological or neurological disease and had normal general and neurological examinations. All subjects were right handed according to the Edinburgh Handedness Inventory (Oldfield, 1971). The protocol was approved by the Ethics Committee of the University Hospital, and all subjects gave their written informed consent for the study, according to the declaration of Helsinki, after its nature was fully explained to them.

Neurophysiological study. Steady-state, auditory-evoked potentials during wakefulness were recorded at 21 scalp locations (10-20 EEG international system); all referred to a binaural reference. Electrode impedance was kept at $<5000 \Omega$. The stimuli were click applied monoaurally to the right ear at the suprathreshold intensity of $80 \mathrm{~dB}$ HL (Picton et al., 1987) and at 12 different frequencies (12, 20, 30, 32.5, 35, 37.5, 40, 42.5, $45,47.5,50$, and $60 \mathrm{~Hz}$ ). Responses were bandpass filtered between 1 and $500 \mathrm{~Hz}$. Five hundred responses, in epochs of $500 \mathrm{msec}$, were averaged for each stimulus frequency. At the 21 electrodes, a fast Fourier transform (FFT) was performed in each trial. The dominant frequencies of power spectra [stimulus rate locked responses (SRLRs)] were determined for all stimulation frequencies, and the amplitude (square root of power) was obtained at the 21 electrodes. All of the dominant peaks of frequency and harmonic and subharmonic responses were measured. In addition, for each stimulation frequency, we measured the $40 \mathrm{~Hz}$ component of the elicited response. This neurophysiological study identified F3 as the electrode where maximum amplitude steady-state evoked potentials were recorded, and therefore, we selected electrical activities at F3 to compare them with rCBF PET measurements (see Fig. 1).

PET procedure. In nine subjects of the group (four females; five males; 
mean age $=30.4 ; \mathrm{SD}=4.8$ ), selected on the basis of availability and whose EEG data were no different from the entire group of 28 subjects, we measured $\mathrm{rCBF}$ with PET- $\mathrm{H}_{2}{ }^{15} \mathrm{O}$ at rest and during auditory stimulation at four different frequencies: $12,32,40$, and $47 \mathrm{~Hz}$. The PET scans were performed with an ECAT EXACT HR+ (Siemens/CTI, Knoxville, TN) that collected 63 simultaneous parallel planes over a $15.2 \mathrm{~cm}$ axial field of view. The tomographic resolution was $4.5 \mathrm{~mm}$. Transmission scanning was done before radiopharmaceutical injection using three ${ }^{68} \mathrm{Ge}$ rotating rod sources. The subject was positioned so that the entire intracranial volume, including the cerebellum, was included in the fields of view.

The subjects lay comfortably in a supine position. No attempt was made to control the subjects' thought content or attention. A small catheter was placed in the left cubital vein for the injection of the radioisotope. Auditory stimulation was performed through a miniearphone introduced in the right external auditory canal. The left external auditory canal was occluded with a silicone stopper. Series of nonfiltered clicks were applied monoaurally to the right ear, at $80 \mathrm{~dB}$ intensity. Each subject underwent five consecutive scans at $20 \mathrm{~min}$ intervals, one at rest and one for each frequency of auditory stimulation $(12,32,40$, and $47 \mathrm{~Hz})$. The order of the different frequency stimulation and baseline scans was randomized across subjects to avoid an order effect. For the baseline scan, subjects lay quietly. After $60 \mathrm{sec}$ at rest or listening to clicks, subjects received $370 \mathrm{MBq}$ of $\mathrm{H}_{2}{ }^{15} \mathrm{O}$ as an intravenous bolus. Scans were initiated automatically when the radioactive count rate in the brain reached a threshold value of $100 \mathrm{kcounts} / \mathrm{sec}, \sim 20 \mathrm{sec}$ after intravenous injection, and continued for $60 \mathrm{sec}$. Emission data were corrected for attenuation by means of the transmission scan performed previously.

PET scans were analyzed using Statistical Parametric Mapping (SPM99; Wellcome Department of Cognitive Neurology, London, UK) (Friston et al., 1995b, 1996) on a Matlab 5.3 platform (MathWorks, Natick, MA). Head movement was corrected by rigid alignment (Friston et al., 1995a). The scans were then spatially normalized using the template from the Montreal Neurological Institute series and the reference system of Talairach and Tournoux (1988). The scans were smoothed using a Gaussian filter set at $10 \mathrm{~mm}$ full width at half-maximum in plane to increase the signal-to-noise ratio. Data were analyzed after construction of a design matrix for the analysis of group data for conditions and covariates. All scans were subjected to an analysis of covariance (ANCOVA). This procedure removes the confounding effect of differences in global activity across scans and normalizes global activity (measured as radioactive counts) to a notional mean $\mathrm{rCBF}$ of 50 $\mathrm{ml} \cdot \mathrm{dl}^{-1} \cdot \min ^{-1}$. For each voxel, the ANCOVA generated five conditionspecific mean $\mathrm{rCBF}$ values and associated error variances.

With SPM, we performed a cognitive subtraction, comparing the mean blood flow elicited by the different stimulation frequencies on a voxel-tovoxel basis. To make inferences about stimulus-frequency-dependent rCBF responses and thereby test our hypotheses, we specified a series of contrasts pertaining to the condition-specific effects, as follows: (1) we compared rCBF auditory-evoked responses across all frequencies relative to rest; we used the ensuing (T) statistical parametric map (from which we derived $Z$ scores only for tabular reporting) to identify the auditory cortex region showing the greatest rCBF response. (2) To test the hypothesis that $40 \mathrm{~Hz}$ stimulation, compared with the other frequencies, may specifically activate regions other than the auditory cortex, we performed a second contrast comparing stimulation at $40 \mathrm{~Hz}$ with the group of the other repetitive stimulation frequencies; we used the ensuing $(\mathrm{T})$ statistical parametric map to identify the cerebellar region showing the greatest $\mathrm{rCBF}$ response. (3) To assess the correlation between the EEG response and the $\mathrm{rCBF}$ activation at the voxels with the greatest activation, determined from the two previous contrasts (in the auditory area and cerebellum), we then specified a third contrast. Its weights were obtained by linear interpolation for the frequencies used in the PET experiment from the amplitude of the EEG SSRs, using the data presented in Figure $1 B$. The resulting four amplitudes were then mean corrected to a mean of 0 , and the rest condition was discounted using a contrast weight of 0 . The contrast testing the hypothesis that the EEG steady-state response could predict activation was $0,-0.8,0.6,2.1$, and -1.9. It is important to note that this contrast is orthogonal with each of the two previous contrasts applied (1 and 2$)$. In other words, the identification of the most responsive auditory voxel is independent of frequency-specific effects.

We used an uncorrected threshold of $p<0.001$. Because our inferences were restricted to the cerebral and cerebellar auditory areas, this

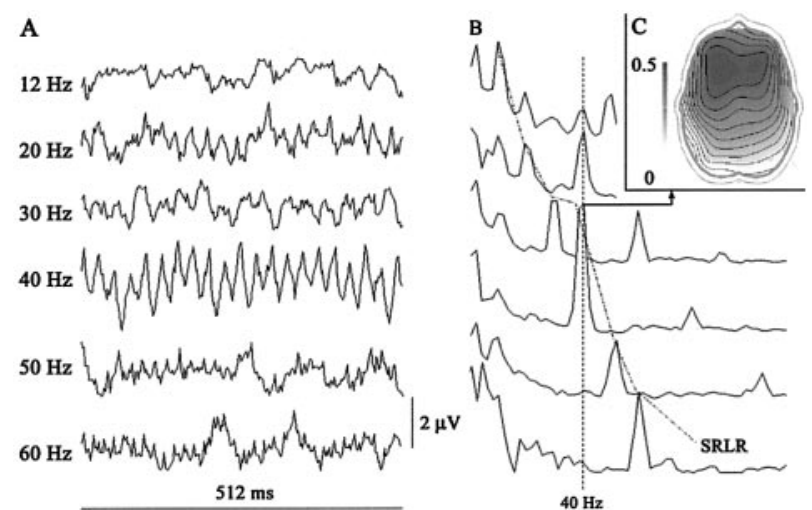

Figure 1. A, B, Electroencephalographic recording at electrode F3 of steady-state auditory potentials from a normal subject receiving auditory stimuli at the following frequencies: $12,30,40,50$, and $60 \mathrm{~Hz}$. A, Steadystate responses; average of 500 trials. $B$, Grand average of steady-state fast Fourier transform at 12, 20,30,40,50, and $60 \mathrm{~Hz}$ stimulation frequencies. Note the peaks at $40 \mathrm{~Hz}$ when the stimulation frequency was at one of its subharmonic rates, 10 and $20 \mathrm{~Hz}$ ( $40 \mathrm{~Hz}$ label), and the peaks at the rate of auditory stimulation (SRLR). $C$, EEG map showing the topography of the $40 \mathrm{~Hz}$ SRLR grand average of the entire experimental group.

corresponds to a threshold of $p<0.05$ correcting for the volume of interest.

\section{RESULTS}

\section{Neurophysiological study}

The steady-state, auditory-evoked response, phase locked with the presented click frequency, was recorded at 12, 20, 30, 32.5, 35, $37.5,40,42.5,45,47.5,50$, and $60 \mathrm{~Hz}$. As a result of a rapidly repeated auditory stimulus application, an initial transient response evolved into an oscillatory EEG response having the same frequency as the stimulus (SRLR). Figure $1 A$ represents average recordings of the steady-state, auditory-evoked responses in a normal subject at the different frequencies. The oscillatory response reached the greatest amplitude at $\sim 40 \mathrm{~Hz}$ and subsequently decreased at higher click rates. Figure $1 B$ represents the grand average of steady-state fast FFTs at 12, 20, 30, 40, 50, and $60 \mathrm{~Hz}$ stimulation frequencies. The fast Fourier transforms analysis revealed three major components. A first component peaked at $10 \mathrm{~Hz}$ and had an occipital predominance. It is possibly related to EEG $\alpha$ activity. A second component peaked at the rate of auditory stimulation and was time locked with the stimulus rate (SRLR). Its amplitude (square root of the power) depended on the stimulation frequency, reaching a maximum at $\sim 40 \mathrm{~Hz}$ (37.75; SD of 1.84) (Fig. 2). We extracted the mean value at 12 , 32,40 , and $47 \mathrm{~Hz}$ for the group of nine subjects who had PET to compare them with $\mathrm{rCBF}$ values $(12 \mathrm{~Hz}=22.23 ; 32 \mathrm{~Hz}=35.4$; $40 \mathrm{~Hz}=50.59 ; 47 \mathrm{~Hz}=10.99)$. The third component was observed at $40 \mathrm{~Hz}$ when the stimulation frequency was at one of its subharmonic rates, 10 and $20 \mathrm{~Hz}$.

Because we used binaural reference electrodes, the largest positive and negative potentials of the steady-state, auditoryevoked responses at $40 \mathrm{~Hz}$ were recorded in the frontal region contralateral to the side stimulated (at the F3 electrode with right ear stimulation) (Fig. $1 C$ ). For this reason, in all subjects, we chose the activity recorded at F3 to compare it with $\mathrm{rCBF}$ changes.

\section{PET studies}

Compared with rest, repetitive auditory stimulation of the right ear at $12,32,40$, and $47 \mathrm{~Hz}$ elicited an increase in rCBF, shown 


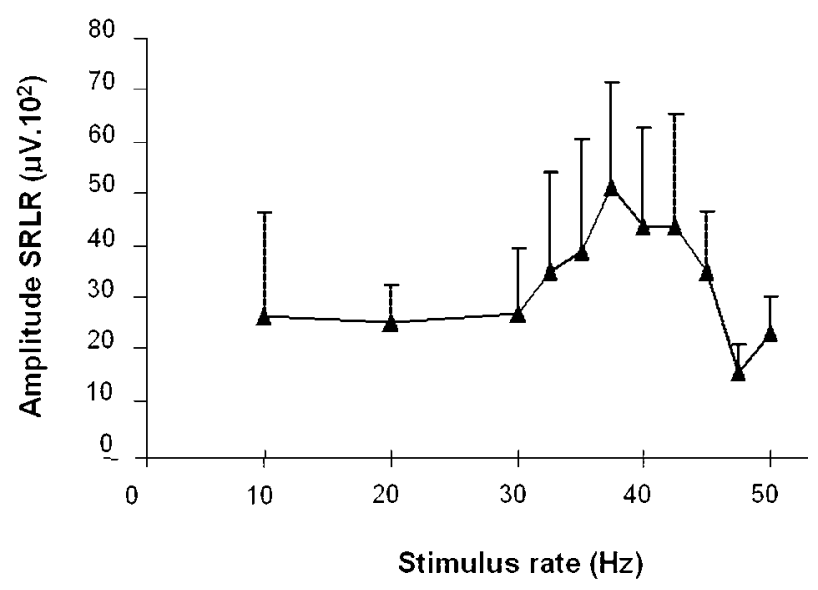

Figure 2. Effect of the auditory stimulation at different rates on the amplitude of the SRLR (grand average of 28 normal subjects). The amplitude (square root of the power of the fast Fourier transforms) of the SRLR reached a maximum at $\sim 40 \mathrm{~Hz}(37.75$; SD of 1.84). Error bars indicate SD.

on SPM(T) maps, in two larger areas: (1) contralateral auditory cortex (Heschl's gyrus) and (2) contralateral superior temporal gyrus and three smaller areas: (3) ipsilateral postcentral gyrus, (4) ipsilateral inferior temporal gyrus, and (5) ipsilateral posterior Sylvian area (Table 1, Fig. 3). A decrement in rCBF was observed at the head of the contralateral caudate nucleus and ipsilateral posterior cingulate cortex (Table 1, Fig. 3). The changes in $\mathrm{rCBF}$ in the auditory area followed a similar pattern to that of the EEG SRLRs at the different frequencies, with the highest activation at $40 \mathrm{~Hz}$.

Compared with stimulation at other frequencies, $40 \mathrm{~Hz}$ stimulation elicited an increased $\mathrm{rCBF}$ in the cortex of the posterior aspect of both cerebellar hemispheres, predominantly on the side contralateral to auditory stimulation (Table 2, Fig. 4). The activated area, lateral to the paravermian region, was located on crus II using Schmahmann's nomenclature (Schmahmann et al., 1999). At the voxel maximally activated in these areas, rCBF clearly peaked during $40 \mathrm{~Hz}$ stimulation compared with other stimulation frequencies (Fig. 4).

In the third contrast, derived from the SRLR values, both the temporal auditory cortex and cerebellar auditory clusters showed a pattern of $\mathrm{rCBF}$ activation similar to that of the EEG SRLRs at the different frequencies, with the highest activation induced by $40 \mathrm{~Hz}$. However, although all stimulation frequencies increased $\mathrm{rCBF}$ at the temporal cortex, stimulation frequencies other than $40 \mathrm{~Hz}$ actually depressed or failed to significantly change rCBF at the cerebellar clusters. The difference can be seen comparing Figure 3 (the parameter estimates at the temporal cortex cluster) with Figure 4 (the parameter estimates at the cerebellar clusters).

\section{DISCUSSION}

\section{Neurophysiological study}

The results of our electrophysiological study agree with those of Galambos et al. (1981) and Azzena et al. (1995). The SRLRs increased in amplitude in the $30-40 \mathrm{~Hz}$ range and decreased at rates of $>40 \mathrm{~Hz}$. The topography of the EEG steady-state response in our study, with greatest amplitude in contralateral frontal electrodes, is a standard finding when the recording is performed with balanced earlobe reference electrodes (Azzena et al., 1995; Maiste et al., 1995). Using a noncephalic reference, the recordings show phase reversals in temporal regions (Johnson et al., 1988). This tangential dipole is better defined by magnetoencephalography (MEG) studies, which demonstrate the source in the primary auditory cortex, with a projection to central fields (Engelien et al., 2000). Because the amplitude of the EEG response obtained with our study design was greatest at F3, it seemed logical to use data from this electrode to compare them with $\mathrm{rCBF}$ data.

\section{PET studies}

For the study of auditory stimuli, PET has advantages compared with functional magnetic resonance imaging (fMRI). PET systems are quiet, whereas the radio frequency generators in MRI units produce noise that can reach $117 \mathrm{~dB}$, thus masking study stimuli (Counter et al., 1997). Although PET systems do not have as much spatial resolution as MRI, their spatial resolution is superior to that of most neurophysiological systems and is not restricted to the study of cortical structures.

\section{Auditory stimulation compared with baseline}

Activated clusters were located in the contralateral primary and secondary auditory cortex, in the contralateral caudate nucleus, and in the ipsilateral posterior cingulate cortex. With the first contrast applied, all frequencies versus rest, the cerebellar hemispheres did not appear to be activated. However, only clusters of cerebellar activation appeared in the second contrast, when we compared $40 \mathrm{~Hz}$ versus the other stimulation frequencies. The difference between the results of the two contrasts is explained by the results of the third contrast. Both the temporal auditory cortex and the cerebellar auditory clusters showed a pattern of $\mathrm{rCBF}$ activation similar to that of the EEG SRLRs at the different frequencies, with the highest activation induced by $40 \mathrm{~Hz}$. However, although all stimulation frequencies increased $\mathrm{rCBF}$ at the temporal cortex, stimulation frequencies other than $40 \mathrm{~Hz}$ actually depressed or failed to significantly change $\mathrm{rCBF}$ at the cerebellar clusters. Thus, the cerebellar cluster did not appear when the group of all frequencies was compared with rest, because the negative effect of some of the frequencies cancelled out the rCBF-enhancing effect of $40 \mathrm{~Hz}$ stimulation (compare Fig. 3 with Fig. 4).

\section{Auditory cortex}

We found an asymmetry in temporal lobe activation, with a larger $\mathrm{rCBF}$ increase in the region of the contralateral primary auditory cortex. Studies using monaural auditory stimulation have shown a strong contralateral temporal lobe activation (Hirano et al., 1997). We also found a second smaller activated area in the superior temporal gyrus, surrounding the primary auditory cortex. This area has been enhanced in studies using complex auditory patterns of stimulation, such as music or speech, suggesting that it may have a role in temporal auditory pattern detection (Creutzfeldt and Ojemann, 1989; Zatorre et al., 1992).

SSRs show maximum amplitude when tone pulses are presented at repetition rates near $40 \mathrm{~Hz}$. To explain this finding, it has been postulated that the SSR consists of superimposed transient middle latency responses that display wave periods near 40 $\mathrm{Hz}$ and summate with one another when phase locked by $40 \mathrm{~Hz}$ steady-state stimulation. Some neurophysiologial data, however, seemed to contradict this postulate. Using tones and MEGrecording techniques, Pantev et al. (1996) studied the cortical sources of the $40 \mathrm{~Hz}$ auditory steady-state fields (SSFs) and middle latency auditory-evoked potentials. They found that these 
Table 1. Cerebral regions activated (rCBF) comparing the effect of grouping auditory repetitive stimulation at all frequencies versus the condition at rest

\begin{tabular}{lcccc} 
Type of rCBF change and area activated & $k$ & $t$ & $Z$ & $p, y, z$ \\
\hline rCBF increment & & & & \\
$\quad$ Contralateral auditory area (left) & 515 & 9.38 & 5.96 & $<0.0001$ \\
$\quad$ Contralateral superior temporal gyrus (left) & 122 & 5.21 & 4.19 & $<0.0001$ \\
$\quad$ Ipsilateral postcentral gyrus (right) & 35 & 4.14 & 3.55 & $<0.0001$ \\
$\quad$ Ipsilateral inferior temporal gyrus (right) & 31 & 3.99 & 3.44 & $<0.0001$ \\
$\quad$ Ipsilateral posterior Sylvian area (right) & 21 & 4.47 & 3.75 & $<0.0001$ \\
rCBF decrement & & & $36,-26,10$ \\
$\quad$ Contralateral caudate (left) & 111 & 4.45 & $36,-30,-20$ \\
Ipsilateral posterior cingulate cortex (right) & 77 & 5.56 & 4.38 & $<0.0001$ \\
\hline
\end{tabular}

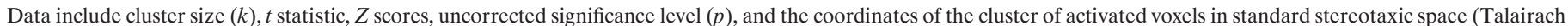
and Tournoux, 1988) $(x, y, z)$.
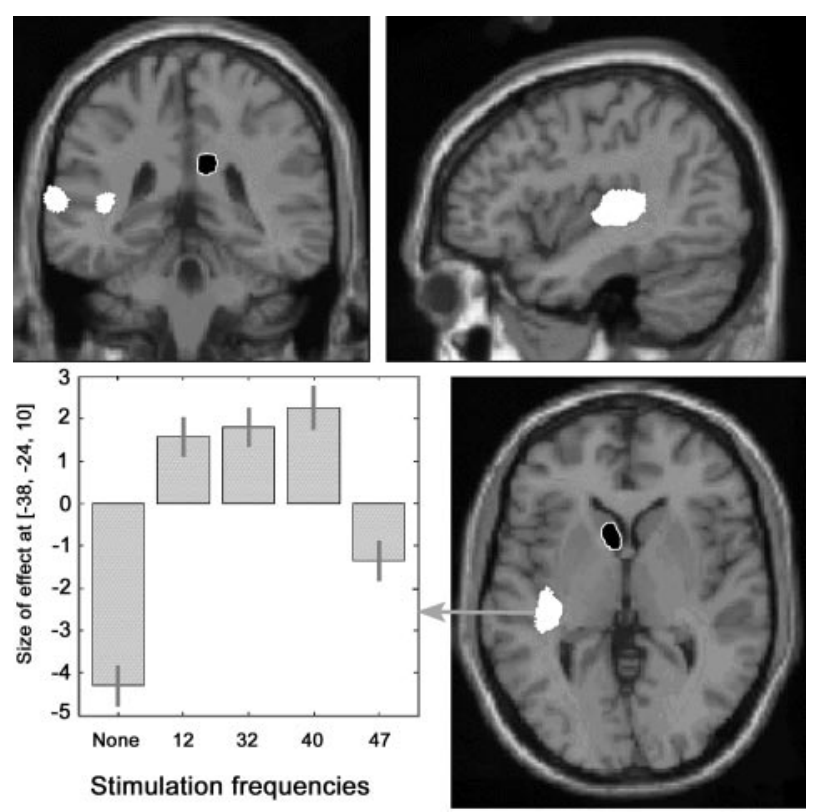

Figure 3. Anatomical location of areas with significant changes in $\mathrm{rCBF}$ during auditory stimulation (grouping of all frequencies compared with resting condition) projected on coronal, sagittal, and transverse sections of a T1-weighted canonical MRI of the human brain (increment in white and decrement in black with white margin). Data are given in Table 1. The chart depicts the size of the effect of the parameter estimates weighted by the amplitude of the EEG steady-state auditory responses in the voxel of greatest activation $(x,-36 ; y,-26 ; z, 10)$ at rest and at each of the four stimulation frequencies.

two types of responses arise in different locations at the auditory area, suggesting a diverse origin for the $40 \mathrm{~Hz}$ SSF and entrained middle latency responses. However, direct evidence of increased temporal cortex synaptic activity specific to $40 \mathrm{~Hz}$ stimulation was lacking. We worked with the hypothesis that rCBF in the auditory area of the temporal lobe would follow a similar pattern to the amplitude of the electrical steady-state response, increasing as the stimulus rate climbed to $30-40 \mathrm{~Hz}$ and decreasing at higher rates. The results support our hypothesis: the rCBF in the voxel with the greatest activation of the cluster located in the auditory cortex increased at auditory stimulation rates from 12 to $40 \mathrm{~Hz}$, decreasing at $47 \mathrm{~Hz}$. This finding suggests that the enhanced EEG response to stimulation at $40 \mathrm{~Hz}$ is not just the result of increased neuronal synchronization but reflects an overall increase in auditory cortex synaptic activity at this frequency. Thus, our study contributes to clarification of an ongoing controversy regarding the origin of the steady-state potentials (Basar et al., 1987; Santarelli et al., 1995; Gutschalk et al., 1999).

\section{Region of the head of the left caudate nucleus and right} posterior cingular cortex

Compared with rest, repetitive auditory stimulation produced a significant decrement in $\mathrm{rCBF}$ at the head of the left caudate nucleus and right posterior cingulate cortex. The caudate is activated to a similar degree by auditory stimulation during wakefulness and non-rapid-eye-movement sleep (Portas et al., 2000). Thus, even a stimulation paradigm such as ours, not specifically requiring any attention or any other perceptual or cognitive effort, is likely to activate the caudate nucleus. There is ample evidence that this structure participates in the processing of more complex auditory tasks. For instance, target detection of auditory stimuli activates the caudate nuclei and posterior cingulate regions (Kiehl et al., 2001). Posterior cingulate gyrus activation has been described even with simple auditory stimulation paradigms, and a decrement in $\mathrm{rCBF}$ occurred with high-intensity stimuli, similarly to what happened in our study (Lockwood et al., 1999).

\section{Activation by $40 \mathrm{~Hz}$ compared with other stimulation frequencies}

Compared with stimulation at lower or higher frequencies, auditory stimulation at $40 \mathrm{~Hz}$ caused bilateral activation of the cerebellar hemispheres, with some contralateral dominance. The activated area was in the posterolateral portion of the hemisphere, lateral to the paravermian region, in crus II using Schmahmann's nomenclature (Schmahmann et al., 1999). A similar location in the cerebellum was activated in other PET studies exploring temporal auditory processing (Penhune et al., 1998; Lockwood et al., 1999; Griffiths, 2000; Ramnani et al., 2000). The anatomical coincidence emphasizes the important role of this cerebellar region in the processing of information related to auditory stimuli. This region differs from the vermian and floccular areas that receive direct cochlear and collicular input. It corresponds to an area receiving auditory, visual, and somesthetic information. Connectivity studies, performed primarily in the cat and in rodents, have determined that the main bulk of afferents to this area originates in the temporal lobe and has a relay in the pontine nuclei before reaching the cerebellar cortex. In the primate, Schmahmann and Pandya (1991) found that projections from the primary auditory area are lacking. Instead, corticopontine auditory fibers originate in the second auditory area AII and adjacent 


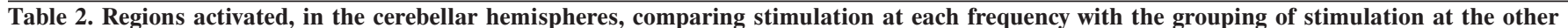
frequencies $(12,32,40$, and $47 \mathrm{~Hz})$

\begin{tabular}{|c|c|c|c|c|c|}
\hline Stimulation frequency & $k$ & $t$ & $Z$ & $p$ & $x, y, z$ \\
\hline $12 \mathrm{~Hz}$ & & & & NS & \\
\hline $32 \mathrm{~Hz}$ & & & & NS & \\
\hline \multicolumn{6}{|l|}{$40 \mathrm{~Hz}$} \\
\hline Contralateral cerebellar hemisphere (left) & 54 & 3.95 & 3.41 & 0.0001 & $-30,-82,-42$ \\
\hline Ipsilateral cerebellar hemisphere (right) & 16 & 3.75 & 3.28 & 0.001 & $28,-80,-48$ \\
\hline $47 \mathrm{~Hz}$ & & & & NS & \\
\hline
\end{tabular}

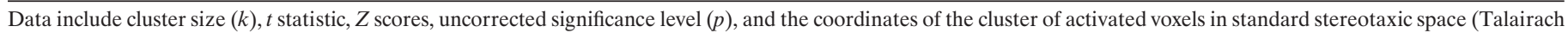
and Tournoux, 1988) $(x, y, z)$.

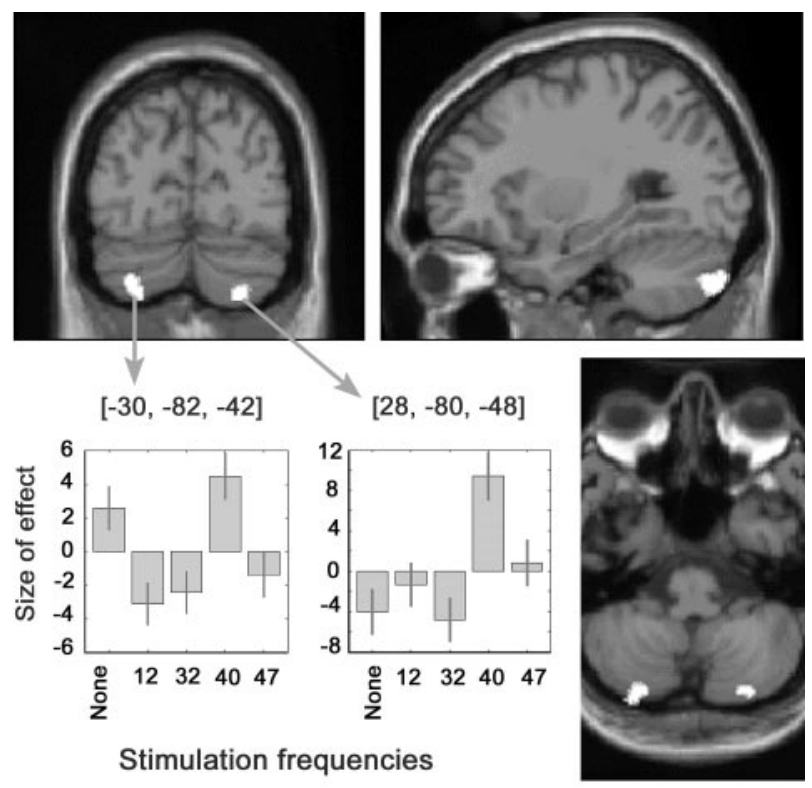

Figure 4. Regions with increased rCBF during $40 \mathrm{~Hz}$ auditory stimulation compared with stimulation at other frequencies. The activated areas, larger in the cerebellar hemisphere contralateral to the stimulated ear, are projected on a canonical image of the human brain obtained with T1weighted MRI in the coronal, sagittal, and transverse planes. The chart depicts the effect size of the parameter estimates weighted for the amplitude of the EEG steady-state auditory response in the voxels of greatest activation $(x,-30 ; y,-82 ; z,-42$; and $x, 28 ; y,-80 ; z,-48)$ at rest and during stimulation at each of the four frequencies. The left cerebellar hemisphere is on the left of the image.

association areas, but the most important bulk of corticopontocerebellar afferents is from multimodal areas in the upper bank of the superior temporal sulcus. These neurons project to the dorsolateral and lateral nuclei of the pons, which, in turn, project to the cerebellar area activated in our study (Brodal, 1979).

It could be argued that cerebellar activation by $40 \mathrm{~Hz}$ stimuli simply represents an enhancement of the spontaneous baseline frequency discharge of Purkinje neurons, from 30 to $50 \mathrm{~Hz}$ (Strahlendorf et al., 1984). Although possible, this explanation does not seem likely, because this baseline frequency is found in the Purkinje neurons of the entire cerebellar cortex and is not restricted to the area activated in our study. Examples include neurons placed more superiorly in the cerebellar hemisphere, with a somatosensory receptive field (Fu et al., 1997), floccular neurons processing vestibular-oculomotor interactions (Fukushima et al., 1999), or visually responsive Purkinje neurons located higher in the cerebellar hemisphere (Marple-Horvat et al., 1998).
To explain why auditory cortex was activated by all stimulation frequencies, whereas cerebellar activation was only detected comparing $40 \mathrm{~Hz}$ with the other frequencies, we could postulate that the cerebellum becomes more active to inhibit excessive cortical firing at some stimulation frequencies. For auditory stimuli, the critical stimulation frequency seems to be $\sim 40 \mathrm{~Hz}$. Perhaps, this is an indirect indication of the propensity of some brain regions to resonate at this frequency (Kapoor et al., 1991). Widespread cortical synchronization at the gamma band, $\sim 40 \mathrm{~Hz}$, may precede photically induced seizures (Parra et al., 2001). Other data suggest a cortical inhibitory role for the cerebellum. Small amplitude electrical stimulation of the cerebellar cortex in humans reduces cortical excitability (Ugawa et al., 1991). Some patients with cortical myoclonus have predominantly cerebellar pathology, suggesting that the enhanced cortical excitability may arise from deficient cerebellar control (Artieda and Obeso, 1993; Tijssen et al., 2000).

Both the neurophysiological studies confirmed by our data and the novel findings of auditory cortex synaptic activation and of specific cerebellar activation at $40 \mathrm{~Hz}$ suggest that this frequency plays a distinct role in the brain mechanisms involved in auditory processing.

\section{REFERENCES}

Artieda J, Obeso JA (1993) The pathophysiology and pharmacology of photic cortical reflex myoclonus. Ann Neurol 34:175-184.

Azzena GB, Conti G, Santarelli R, Ottaviani F, Paludetti G, Maurizi M (1995) Generation of human auditory steady-state responses (SSRs). I. Stimulus rate effects. Hear Res 83:1-8.

Basar E, Rosen B, Basar-Eroglu C, Greitschus F (1987) The associations between $40 \mathrm{~Hz}-\mathrm{EEG}$ and the middle latency response of the auditory evoked potential. Int J Neurosci 33:103-117.

Brodal P (1979) The pontocerebellar projection in the rhesus monkey: an experimental study with retrograde axonal transport of horseradish peroxidase. Neuroscience 4:193-208.

Counter SA, Olofsson A, Grahn HF, Borg E (1997) MRI acoustic noise: sound pressure and frequency analysis. J Magn Reson Imaging 7:606-611.

Creutzfeldt O, Ojemann G (1989) Neuronal activity in the human lateral temporal lobe. III. Activity changes during music. Exp Brain Res 77:490-498.

Engelien A, Schulz M, Ross B, Arolt V, Pantev C (2000) A combined functional in vivo measure for primary and secondary auditory cortices. Hear Res 148:153-160.

Friston KJ, Ashburner J, Frith C, Poline J, Heather J, Frackowiak R (1995a) Spatial registration and normalization of images. Hum Brain Mapp 2:165-189.

Friston KJ, Holmes AP, Worsley KJ, Poline JP, Frith CD, Frackowiak RSJ (1995b) Statistical parametric maps in functional imaging: a general linear approach. Hum Brain Mapp 2:189-210.

Friston KJ, Holmes A, Poline JB, Price CJ, Frith CD (1996) Detecting activations in PET and fMRI: levels of inference and power. NeuroImage 4:223-235.

Fu QG, Flament D, Coltz JD, Ebner TJ (1997) Relationship of cerebellar Purkinje cell simple spike discharge to movement kinematics in the monkey. J Neurophysiol 78:478-491.

Fukushima K, Fukushima J, Kaneko CR, Fuchs AF (1999) Vertical 
Purkinje cells of the monkey floccular lobe: simple-spike activity during pursuit and passive whole body rotation. J Neurophysiol 82:787-803.

Galambos R, Makeig S, Talmachoff PJ (1981) A 40-Hz auditory potential recorded from the human scalp. Proc Natl Acad Sci USA 78:2643-2647.

Griffiths TD (2000) Musical hallucinosis in acquired deafness: phenomenology and brain substrate. Brain 123:2065-2076.

Gutschalk A, Mase R, Roth R, Ille N, Rupp A, Hahnel S, Picton TW, Scherg M (1999) Deconvolution of $40 \mathrm{~Hz}$ steady-state fields reveals two overlapping source activities of the human auditory cortex. Clin Neurophysiol 110:856-868.

Hirano S, Naito Y, Okazawa H, Kojima H, Honjo I, Ishizu K, Yenokura Y, Nagahama Y, Fukuyama H, Konishi J (1997) Cortical activation by monaural speech sound stimulation demonstrated by positron emission tomography. Exp Brain Res 113:75-80.

Johnson BW, Weinberg H, Ribary U, Cheyne DO, Ancill R (1988) Topographic distribution of the $40 \mathrm{~Hz}$ auditory evoked-related potential in normal and aged subjects. Brain Topogr 1:117-121.

Kapoor R, Griffin G, Barrett G, Fowler CJ (1991) Myoclonic epilepsy in an HIV positive patient: neurophysiological findings. Electroencephalogr Clin Neurophysiol 78:80-84.

Kiehl KA, Laurens KR, Duty TL, Forster BB, Liddle PF (2001) Neural sources involved in auditory target detection and novelty processing: an event-related fMRI study. Psychophysiology 38:133-142.

Lockwood AH, Salvi RJ, Coad ML, Arnold SA, Wack DS, Murphy BW, Burkard RF (1999) The functional anatomy of the normal human auditory system: responses to 0.5 and $4.0 \mathrm{kHz}$ tones at varied intensities. Cereb Cortex 9:65-76.

Maiste AC, Wiens AS, Hunt MJ, Scherg M, Picton TW (1995) Eventrelated potentials and the categorical perception of speech sounds. Ear Hear 16:68-90.

Makela JP, Hari R (1987) Evidence for cortical origin of the $40 \mathrm{~Hz}$ auditory evoked response in man. Electroencephalogr Clin Neurophysiol 66:539-546.

Marple-Horvat DE, Criado JM, Armstrong DM (1998) Neuronal activity in the lateral cerebellum of the cat related to visual stimuli at rest, visually guided step modification, and saccadic eye movements. J Physiol (Lond) 506:489-514.

Oldfield RC (1971) The assessment and analysis of handedness: the Edinburgh inventory. Neuropsychologia 9:97-113.

Pantev C, Roberts LE, Elbert T, Ross B, Wienbruch C (1996) Tonotopic organization of the sources of human auditory steady-state responses. Hear Res 101:62-74.
Parra J, Kalitzin S, Blanes W, Kasteleijn-Nolst Trenité D, Lopes da Silva F (2001) Stimulus-induced MEG gamma band synchronization in photosensitive epilepsy. Ann Neurol 50:S47.

Penhune VB, Zattore RJ, Evans AC (1998) Cerebellar contributions to motor timing: a PET study of auditory and visual rhythm reproduction. J Cogn Neurosci 10:752-765.

Picton TW, Vajsar J, Rodriguez R, Campbell KB (1987) Reliability estimates for steady-state evoked potentials. Electroencephalogr Clin Neurophysiol 68:119-131.

Portas CM, Krakow K, Allen P, Josephs O, Armony JL, Frith CD (2000) Auditory processing across the sleep-wake cycle: simultaneous EEG and fMRI monitoring in humans. Neuron 28:991-999.

Ramnani N, Toni I, Josephs O, Ashburner J, Passingham RE (2000) Learning- and expectation-related changes in the human brain during motor learning. J Neurophysiol 84:3026-3035.

Santarelli R, Maurizi M, Conti G, Ottaviani F, Paludetti G, Pettorossi VE (1995) Generation of human auditory steady-state responses (SSRs). II. Addition of responses to individual stimuli. Hear Res 83:9-18.

Schmahmann JD, Pandya DN (1991) Projections to the basis pontis from the superior temporal sulcus and superior temporal region in the rhesus monkey. J Comp Neurol 308:224-248.

Schmahmann JD, Doyon J, McDonald D, Holmes C, Lavoie K, Hurwitz AS, Kabani N, Toga A, Evans A, Petrides M (1999) Threedimensional MRI atlas of the human cerebellum in proportional stereotaxic space. NeuroImage 10:233-260.

Steriade M, Dossi RC, Pare D, Oakson G (1991) Fast oscillations $(20-40 \mathrm{~Hz})$ in thalamocortical systems and their potentiation by mesopontine cholinergic nuclei in the cat. Proc Natl Acad Sci USA 88:4396-4400.

Strahlendorf JC, Lee M, Strahlendorf HK (1984) Effects of serotonin on cerebellar Purkinje cells are dependent on the baseline firing rate. Exp Brain Res 56:50-58.

Talairach J, Tournoux P (1988) Co-planar stereotaxic atlas of the human brain. New York: Thieme.

Tijssen MA, Thom M, Ellison DW, Wilkins P, Barnes D, Thompson PD, Brown P (2000) Cortical myoclonus and cerebellar pathology. Neurology 54:1350-1356.

Ugawa Y, Day BL, Rothwell JC, Thompson PD, Merton PA, Marsden CD (1991) Modulation of motor cortical excitability by electrical stimulation over the cerebellum in man. J Physiol (Lond) 441:57-72.

Zatorre RJ, Evans AC, Meyer E, Gjedde A (1992) Lateralization of phonetic and pitch discrimination in speech processing. Science 256: 846-849. 